\title{
The Etiological Heterogeneity of Bicuspid Aortopathy between Ascending and Root Morphotype
}

Fei Li, MD* ${ }^{* 1,4}$ Xuan Li, MD* ${ }^{1,2}$ Yue-tang Wang, MD,${ }^{1}$ Cun-tao Yu, MD, ${ }^{3}$ Gang Yin, BS, ${ }^{4}$ Xiu-yu Chen, $\mathrm{MD},{ }^{4}$ Shi-hua Zhao, MD, ${ }^{4}$ Wei Wang, $\mathrm{MD},{ }^{1} \mathrm{Qi}$ Gao, $\mathrm{PhD}^{5}$

${ }^{1}$ Department of Structural Heart Disease, Fuwai Hospital, State Key Laboratory of Cardiovascular Disease, Chinese Academy of Medical Sciences and Peking Union Medical College, Beijing, China; ${ }^{2}$ Department of Physiology and Biophysics, University of Mississippi Medical Center, Jackson, MS; ${ }^{3}$ Department of Vascular Surgery, Fuwai Hospital, Chinese Academy of Medical Sciences and Peking Union Medical College, Beijing, China; ${ }^{4}$ Department of Magnetic Resonance Imaging, Fuwai Hospital, Chinese Academy of Medical Sciences and Peking Union Medical College, Beijing, China; ${ }^{5}$ School of Aeronautics and Astronautics, Zhejiang University, Hangzhou, China

\section{ABSTRACT}

Background: Valve-related hemodynamics and intrinsically regulated matrix proteases are 2 determined pathogenetic factors associated with medial elastin degeneration in bicuspid aortopathy. This study analyzed the association between elastic fiber deterioration and the 2 pathogenetic factors in ascending and root morphotypes, aiming to elucidate the etiological heterogeneity between the 2 morphotypes.

Methods: Four-dimensional flow cardiac magnetic resonance was used to measure the regional wall shear stress (WSS) on the ascending aorta, and matrix metalloproteinase (MMP) expression was assessed by immunoblotting. After histopathology analysis of aortic tissue, we assessed whether elevated regional WSS and increased MMP expression corresponded with medial elastin thinning.

Results: Increased regional WSS corresponded with medial elastin thinning in both morphotypes. Increased expression of different MMP isoforms corresponded with medial elastin degeneration in bicuspid aortopathy. The significantly increased expression of MMP-2 corresponded with a decrease of elastic fiber thickness in the ascending morphotype $(\mathrm{P}=.046)$, whereas elastic fiber thinning was associated with high levels of MMP-3 expression $(\mathrm{P}=.012)$ in the root morphotype. No association was observed between regional WSS and MMP expression.

Conclusion: There is no difference in the effect of valverelated hemodynamics between ascending and root morphotype, and MMPs are not involved in the process of elastic

Received September 17, 2020; accepted October 8, 2020.

* F. Lei and X. Li contributed equally to this study.

Correspondence: Shibua Zhao, No. 167 North Lishi Road, Xicheng District, Beijing 100037, China; 8610-88322539 (e-mail: zhaoshibua0202@126.com); Wei Wang, No. 167 North Lishi Road, Xicheng District, Beijing 100037, China; 8610-88396773 (e-mail: drweiwangfuwai@163.com); Qi Gao, No. 38 Zheda Road, Yuquan Campus, Zhejiang University, Xihu District, Hangzhou, 310027 , China; 86571-87952570 (e-mail: qigao@zju.edu.cn). fiber degeneration induced by increased WSS. The increased expression of different MMP isoforms was observed in the context of elastic fiber degeneration between the 2 morphotypes, implying that heterogeneity between them is revealed in the different intrinsic pathway of medial elastin degradation.

\section{INTRODUCTION}

Bicuspid aortic valve (BAV) disease is the most common congenital cardiac abnormality. The prevalence in the general population is between $0.5 \%$ and $2 \%$ [Basso 2004; Fedak 2002]. This disease is usually associated with dilation of the proximal aorta and an increased risk of aortic adverse events such as aortic dissection or rupture [Ward 2000]. Fazel et al [2008] classified the types of proximal aortic dilation according to aortic configuration and recognized bicuspid aortopathy as a heterogeneous disease [Kari 2012]. The heterogeneity is manifested not only as a difference in various configurations of the dilation of proximal aorta, but also, more importantly, as a difference in pathogenesis [Yamashita 2018; Girdauskas 2011].

It is well known that the thinner the medial elastic fiber, the weaker the aortic wall. Valve-related flow turbulence and intrinsic molecular machinery are 2 pathogenetic factors resulting in medial elastin degeneration and leading to the development of bicuspid aortopathy [Longobardo 2016; Guzzardi 2015]. On analysis of the association between aortic configuration and valve hemodynamics (aortic stenosis or aortic regurgitation), some cross-sectional epidemiologic studies [Sievers 2016; Kang 2013; Schaefer 2008] provided preliminary and speculative clues for the etiological heterogeneity of bicuspid aortopathy between ascending and root morphotypes. However, there is currently no experimental evidence to elucidate the heterogeneity between the 2 morphotypes [Girdauskas 2011].

Four-dimensional flow cardiac magnetic resonance (4D flow CMR) can visualize aortic 3D blood flow patterns (e.g., flow jets, vortices, and helical flow) and measure wall shear stress (WSS) on the aortic wall. In this study, we used this 
technique to compute the regional WSS on the ascending aorta. In addition, we evaluated elastic fiber degeneration by pathological analysis. Matrix metalloproteinases (MMPs) are known by their ability to degrade extracellular matrix (ECM) proteins. In the study of Guzzardi et al [2015], MMP-1, -2, and -3 were recognized as the biomarkers involved in ECM degradation and tissue remodeling [Sbardella 2012]. In this study, the protein expression of MMP-1, -2 , and -3 was evaluated by immunoblotting.

To test a hypothesis that in bicuspid aortic valve patients, elastic fiber degeneration causing aortic dilation is associated with valve-related hemodynamics (WSS) or intrinsic molecular machinery (homogeneous or heterogeneous expression of MMPs), we posed the following 3 questions: (1) Does elevated WSS correspond with elastic fiber thinning in the ascending and root morphotypes? (2) Is there a difference in MMP expression between the 2 morphotypes? (3) Do MMPs act as possible mediators in the process of aortic ECM degeneration caused by valve-related hemodynamics? This study aimed to investigate the etiologic heterogeneity of bicuspid aortopathy based on direct experimental evidence.

\section{METHODS}

\section{Study Population}

Approval for this study was granted by the local ethics committee, and the procedures conformed to the principles of the Declaration of Helsinki. Written informed consent was obtained from all participants before enrollment. Thirty BAV patients (ascending morphotype, $\mathrm{n}=15$; root morphotype, $\mathrm{n}=15$ ) who underwent aortic surgery were enrolled between July 2017 and January 2019. Patients with previous cardiac surgery, suspected connective tissue disease, familial aortopathy, or abdominal aortic disease were excluded. Circumferential diameter (maximum) $\geq 40 \mathrm{~mm}$ was defined as aortic dilation at the level of Valsalva sinus and middle ascending aorta [Jackson 2011]. The root morphotype was defined as proximal aortic dilation involving with the ascending aorta and Valsalva sinus, and the ascending morphotype was defined as dilation occurring only in the ascending aorta.

\section{D Flow CMR Image Acquisition and WSS Calculation}

All patients received preoperative CMR examination on a 3T MR scanner (Discovery MR750; GE Healthcare, Waukesha, WI). 4D flow sequence was used for acquiring time-resolved 3D flow velocities. The scan protocol was described in detail in our previous study [Li 2020]. Aortic diameter measurements were obtained from transaxial stacks of half-Fourier-acquisition, single-shot turbo spine-echo scan sequence imaging covering the whole thorax.

4D flow CMR images at the systolic peak were applied from multiple phases in 1 cardiac cycle for the investigation. Image postprocessing included aortic profile extraction, noise reduction, smoothness of velocity field, and calculation and visualization of WSS. All the code for computation was programmed and executed in Matlab software (version R2017b; MathWorks, Natick, MA). Velocity field and magnitude information of 4D flow CMR images were used to extract the profile of the aorta [Huang 1979; Otsu 1979; Kazhdan 2013]. Then, relying on the aortic profile and velocity field, divergence-free smoothing [Wang 2016] with wall treatment was applied to denoise and smooth the interior flow field $[\mathrm{Li}$ 2020]. After acquiring the smoothed velocity field that satisfied divergence-free restraint, a special wall function of the velocity profile based on the vortex-bonding model [Kendall 2008] was modeled to calculate WSS. The average WSS on each aortic region was calculated by using the area integral to WSS in this region, divided by the corresponding area. Tecplot 360 software (Bellevue, WA) was used to visualize the heatmap of WSS.

\section{Ascending Aorta Zoning and Aortic Tissue Collection}

The ascending aorta between the sinus-aortic junction and the origin of the innominate artery was equally divided into 4 isometric parts in the circumferential direction: greater curvature (GC) and lesser curvature (LC) walls and anterior and posterior walls (Figure 1 $A, B$ ). Previous studies [Guzzardi 2015; Bollache 2018] showed that the nonphysiological value of WSS commonly located in the right anterolateral wall of the ascending aorta corresponds to the GC region. Thus, we selected this region and its contralateral wall, corresponding to the $\mathrm{LC}$ region, as a comparison.

For all enrolled patients, tissue strips $(20 \times 10 \mathrm{~mm})$ were harvested from GC and LC regions of the resected ascending aorta. All fresh aortic tissue specimens were washed in phosphate-buffered saline and equally divided into 2 parts. One part was fixed with $10 \%$ neutral buffered formalin for Verhoeff-Van Gieson staining, and the other was immediately preserved in liquid nitrogen for later immunoblotting analysis.
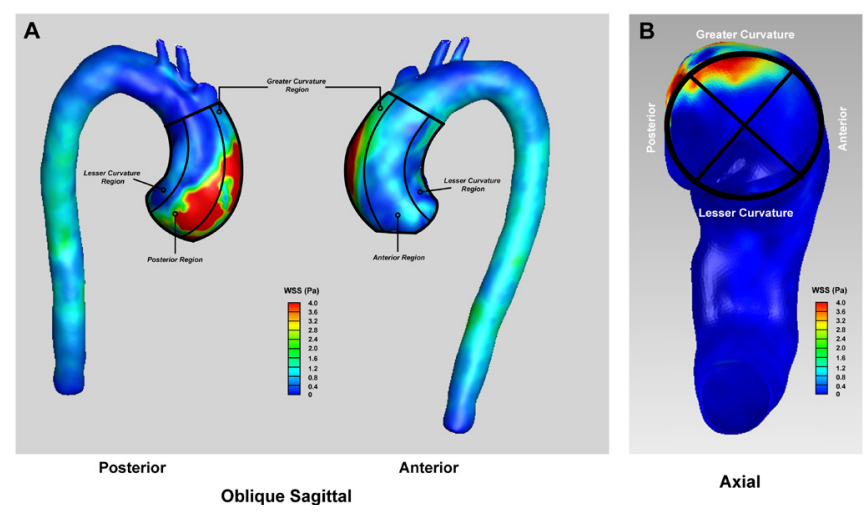

Figure 1. Example of 3D velocity encoding-derived heatmap of WSS of an ascending aorta in a patient and the definition of aortic regions. (A) Heatmap of WSS captured from oblique sagittal images shows that the ascending aorta is equally divided into 4 parts in circumferential direction: greater curvature (GC), lesser curvature (LC), and anterior and posterior walls. (B) Heatmap of WSS captured from axial images shows that the perimeter of the ascending aorta is equally divided to 4 parts to show the definition of aortic regions in circumferential direction. $\mathrm{Pa}=\mathrm{N} / \mathrm{m}^{2}$. 
Table 1. Demographic and Preoperative Characteristics*

\begin{tabular}{|c|c|c|c|}
\hline Male sex & $11(73.3)$ & $14(93.3)$ & .330 \\
\hline Height (cm) & $168.33 \pm 5.96$ & $170.07 \pm 6.26$ & .444 \\
\hline Weight (kg) & $76.00 \pm 13.55$ & $70.47 \pm 12.39$ & .253 \\
\hline Patterns of valvular dysfunction & & & .080 \\
\hline AS & $14(93.3)$ & $9(60.0)$ & \\
\hline AR & $1(6.7)$ & $6(40.0)$ & \\
\hline Sievers phenotype & & & .652 \\
\hline Type-1 RL & $8(53.3)$ & $8(53.3)$ & \\
\hline Type-0 AP & $2(13.3)$ & $1(6.8)$ & \\
\hline \multicolumn{4}{|l|}{ Aortic diameter (mm) } \\
\hline Aortic root & $36.36 \pm 2.71$ & $46.56 \pm 6.20$ & $<.001$ \\
\hline Ascending aorta & $50.44 \pm 5.23$ & $49.39 \pm 8.02$ & .675 \\
\hline Proximal aortic arch & $39.45 \pm 2.90$ & $38.11 \pm 3.48$ & .262 \\
\hline
\end{tabular}

*Categorical variables are expressed as $\mathrm{n}(\%)$; continuous variables are presented as mean \pm SD.

AP indicates anterior-posterior; AR, aortic regurgitation; AS, aortic stenosis; LAT, lateral; LN, left-noncoronary sinus; RL, right-left coronary sinus; RN, right-noncoronary sinus.

\section{Verboeff-Van Gieson Staining}

The aortic tissue sample was fixed for 72 hours at room temperature and embedded in paraffin. Paraffin sections $(5 \mu \mathrm{m})$ prepared from the aortic wall were stained with elastin VerhoeffVan Gieson stain (HT25A-1KT; Sigma-Aldrich, St. Louis, MO) according to the manufacturer's instructions, and each section was imaged under panoramic digital slide scanners (Panoramic SCAN II; 3DHISTECH, Budapest, Hungary). Chromatic analysis of the image was performed with CaseViewer software (version $2.2 ; 3$ DHISTECH) to measure the thickness of elastic fiber (50 measuring points) for each specimen. The multiple measured values were averaged as 1 single value for each enrolled patient.

\section{Immunoblotting Analysis}

Immunoblotting was done as previously described [Zhang 2017]. Briefly, the samples were homogenized and loaded onto Bis-Tris gels (Invitrogen, Carlsbad, CA) and transferred to polyvinylidene fluoride membranes using iBlot 2 Dry Blotting System (Invitrogen). The membrane was then incubated with the appropriate specific primary antibody, MMP-1 (ab38929; Abcam, Cambridge, UK), MMP-2 (ab37150; Abcam), or MMP-3 (ab52915; Abcam), at $4^{\circ} \mathrm{C}$ overnight. It was then treated with horseradish peroxidase-linked secondary antibody at room temperature for 1 hour. Luminescence

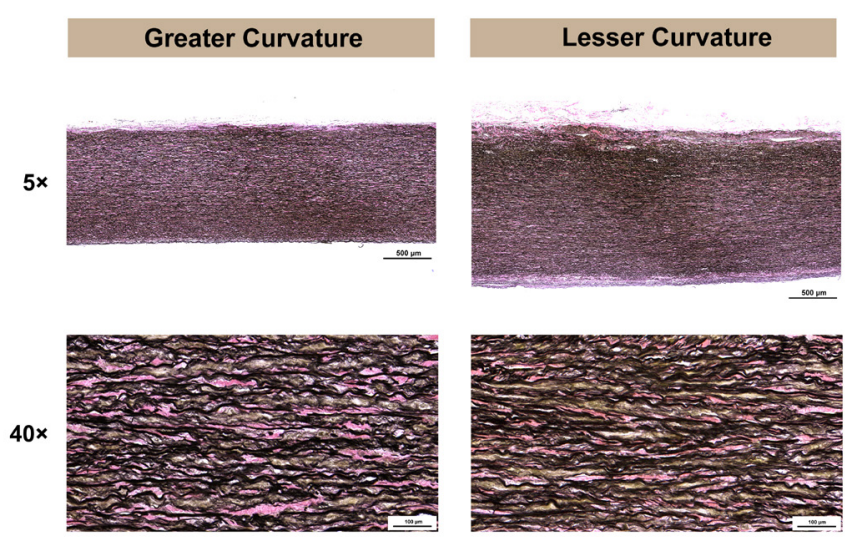

Figure 2. Verhoeff-Van Gieson elastin staining of the aortic wall in GC and $\mathrm{LC}$ regions.

was detected using a chemi-doc image analyzer (FluorChemo M FM0488; Protein Simple, San Jose, CA).

\section{Statistical Analysis}

Continuous variables are reported as mean \pm standard deviation (SD) or median (interquartile range), and categorical 
variables are presented as frequencies and percentages. Shapiro-Wilk test was used to evaluate whether data were normally distributed. Paired Student's $t$ test or Wilcoxon matched-pair signed-rank test or Friedman test with post hoc Bonferroni correction was used to compare the related samples between groups. Mann-Whitney $U$ test or $t$ test was used to compare independent samples between groups. Correlation analyses were performed by Spearman correlation analysis. Fisher's exact test or $\chi^{2}$ test was used to compare frequencies between groups. Statistical analyses were carried out using SPSS version 25 (IBM, Armonk, NY), and 2 -sided $P<.05$ was considered statistically significant.

\section{RESULTS}

\section{Baseline Characteristics}

Preoperative characteristics and demographics of patients are shown in Table 1 . The mean age of all enrolled patients was $54.30 \pm 12.40$ years, and $83.3 \%$ of them $(n=25)$ were male. Seventeen patients $(57.67 \%)$ had hypertension. The mean aortic diameter of all patients was $41.46 \pm 7.00 \mathrm{~mm}$ at Valsalva sinus, $49.92 \pm 6.67 \mathrm{~mm}$ at middle ascending aorta, and $38.78 \pm 3.22$ $\mathrm{mm}$ at proximal aortic arch. The difference of characteristics between the 2 morphotypes, including sex ratio, mean age, incidence of hypertension, patterns of valvular dysfunction, and bicuspid valve phenotype, did not reach statistical significance.

\section{Increased WSS Corresponds with Elastic Fiber Thinning}

The average thickness of elastic fiber in the GC region was significantly thinner than in the LC region in both ascending and root morphotypes of bicuspid aortopathy (both $P<.001$ ) (Table 2 and Figure 2). However, no significant difference was observed when we compared elastic fiber thickness at the same regions (GC or LC) between the 2 phenotypes $(P=.417$ and $P=.308$, respectively) (Table 2 ).
Mean WSSs in the 4 ascending aortic regions were compared first. For all enrolled patients, mean WSS in the GC region was significantly higher than in the other 3 regions (GC versus anterior, $P=.042$; GC versus $\mathrm{LC}, P<.001$; GC versus posterior, $P<.001)$. In addition, the mean WSSs between GC and LC regions were compared in the 2 morphotypes. The mean WSS in the GC region was significantly higher than in the LC region in both morphotypes $(P<.001$ and $P=.028$, respectively) (Table 2). No significant difference was observed in mean WSS at the same region (GC or LC) between the 2 morphotypes ( $P=.967$ and $P=.174$, respectively) (Table 2). Increased regional WSS corresponded with medial elastin thinning in both morphotypes.

\section{Heterogeneity of MMP Expression in 2 Morphotypes}

The expression of MMP-1, -2 , and -3 in aortic tissues was evaluated by Western blotting analysis. In the 2 morphotypes, the trend of MMP expression was completely opposite (Figure $3 A, B)$. Elevated expression of MMP-2 was observed in the GC region compared to the $\mathrm{LC}$ region in patients with ascending morphotype $(P=.046)$ (Figure 3D), but in root morphotype, the expression of MMP-2 in the LC region was slightly higher than in the GC region, without significant difference $(P=.125$; Figure $3 G$ ). In addition, markedly increased expression of MMP-1 and MMP-3 was found in the LC region in the ascending morphotype $(P=.027$ and $P=.001)$ (Figure $3 C, E)$. Intriguingly, the expression of MMP-3 was significantly upregulated in the $\mathrm{GC}$ region in the root morphotype $(\mathrm{P}=.012)$ (Figure $3 H)$. A similar trend was also seen in MMP-1 expression, although the difference was not significant $(\mathrm{P}=.090$; Figure $3 F)$.

\section{Relationship between WSS and MMP Expression}

Correlation was analyzed between regional WSS and MMP expression. Elevated regional WSS and higher expression of MMP-2 was observed in the GC region in BAV patients with ascending morphotype, but no correlation was

Table 2. Regional WSS and Elastic Fiber Thickness*

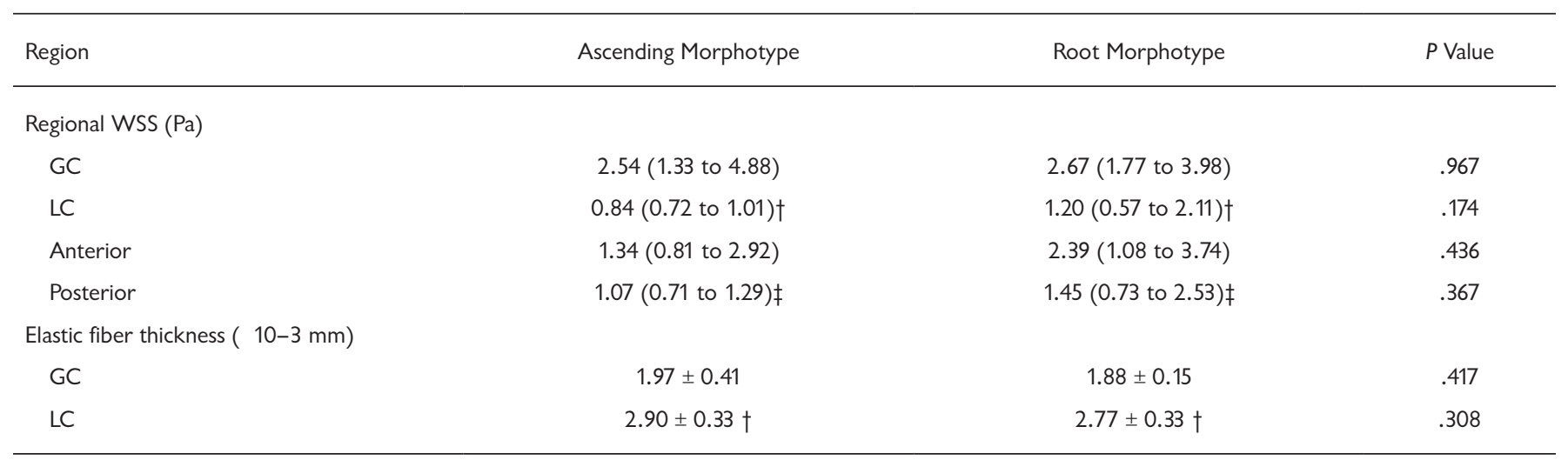

*Continuous variables are reported as mean $\pm \mathrm{SD}$ or median (interquartile range). $\mathrm{P}$ values in the table refer to ascending morphotype versus root morphotype.

$\dagger P<.05, G C$ versus LC.

$\ddagger P<.05, G C$ versus posterior. 
obtained between the 2 factors $(r=0.189, P=.318)$. In addition, regional WSS did not correlate with the expression of MMP-1 or -3 in these patients, either $(r=-0.135, P=.477 ; r=$ $-0.137, P=.469$; respectively). Similarly, in root morphotype, there was no correlation between regional WSS and MMPs 1,2 , or $3(r=-0.281, P=.133 ; r=0.078, P=.683 ; r=-0.053$, $P=.782$; respectively; Figure 4).

\section{DISCUSSION}

Although the etiological heterogeneity of bicuspid aortopathy was speculated in previous studies [Yamashita 2008; Girdauskas 2011; Longobardo 2016], there was no direct experimental evidence. The present study investigated the association between medial elastin thinning, valve-related hemodynamics, and the expression of extracellular matrix proteases, aiming to give further elaboration about the etiological heterogeneity of bicuspid aortopathy between ascending and root morphotypes. Our findings revealed that medial elastin thinning in the ascending and root morphotypes is associated with the activation of different MMP subtypes, indicating that different intrinsically regulated mechanisms of matrix proteases are involved with the process of aortic extracellular remodeling in the 2 morphotypes. Previous epidemiological analysis [Sievers 2016; Kang 2013; Schaefer 2008] speculated that the effect of hemodynamics may be different between the ascending and root morphotypes. However, we found that increased regional WSS corresponded with elastic fiber thinning in both morphotypes, indicating no difference in the effect of valve-related hemodynamics between them.

Nagase et al [1999] demonstrated in detail the function of MMPs and elaborated the theory [Rabkin 2017] that the different types of ECM components are degraded by different MMP isoforms. Based on this theory, our results imply that the process of elastic fiber degradation of bicuspid aortopathy may be involved with different genetically determined intrinsic mechanisms in the 2 morphotypes. However, an aberration in MMP expression was observed, showing that

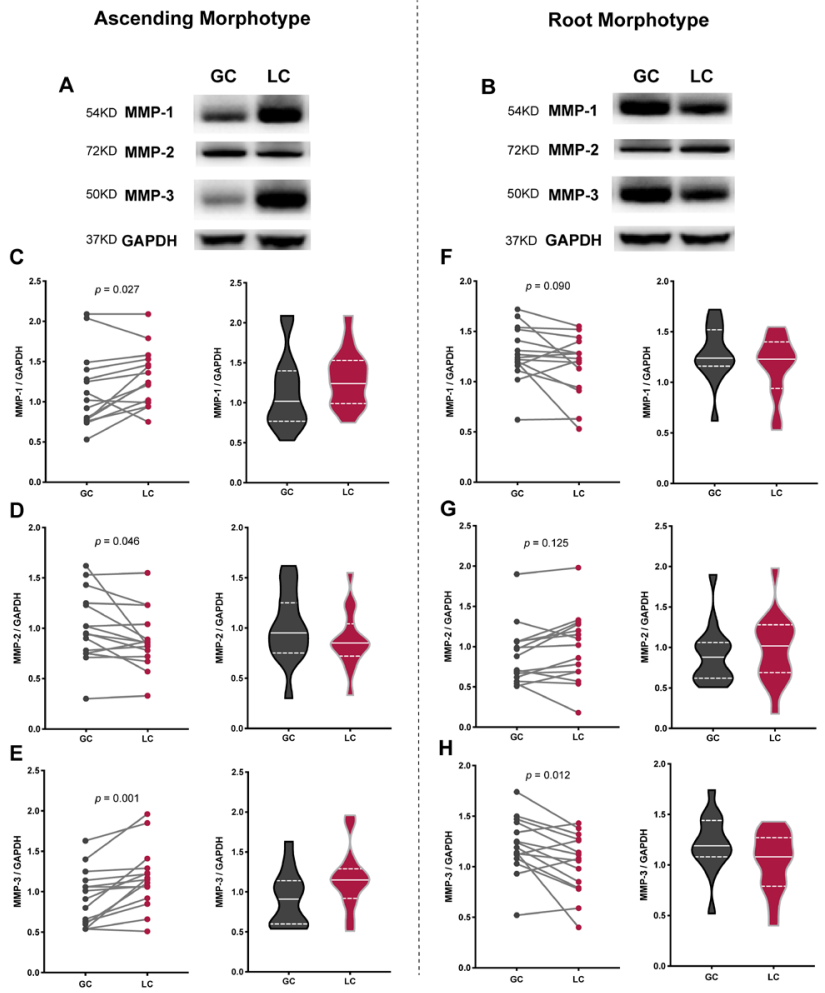

Figure 3. MMP expression in ascending and root morphotypes. ( $\mathrm{A}$ and B) Representative blots of MMPs in ascending and root morphotypes. (C-H) Comparison of MMP expression between GC and LC regions. GAPDH indicates glyceraldehyde 3-phosphate dehydrogenase.

MMP-1 and MMP-3 were dominant on the LC side in the ascending morphotype but on the GC side in the root morphotype, and MMP-2 was dominant on the GC side in the ascending morphotype but on the LC side in the root morphotype. Although these results are stronger evidence to elucidate the etiological heterogeneity between ascending and root morphotype, they are in violation of the dogma, "the
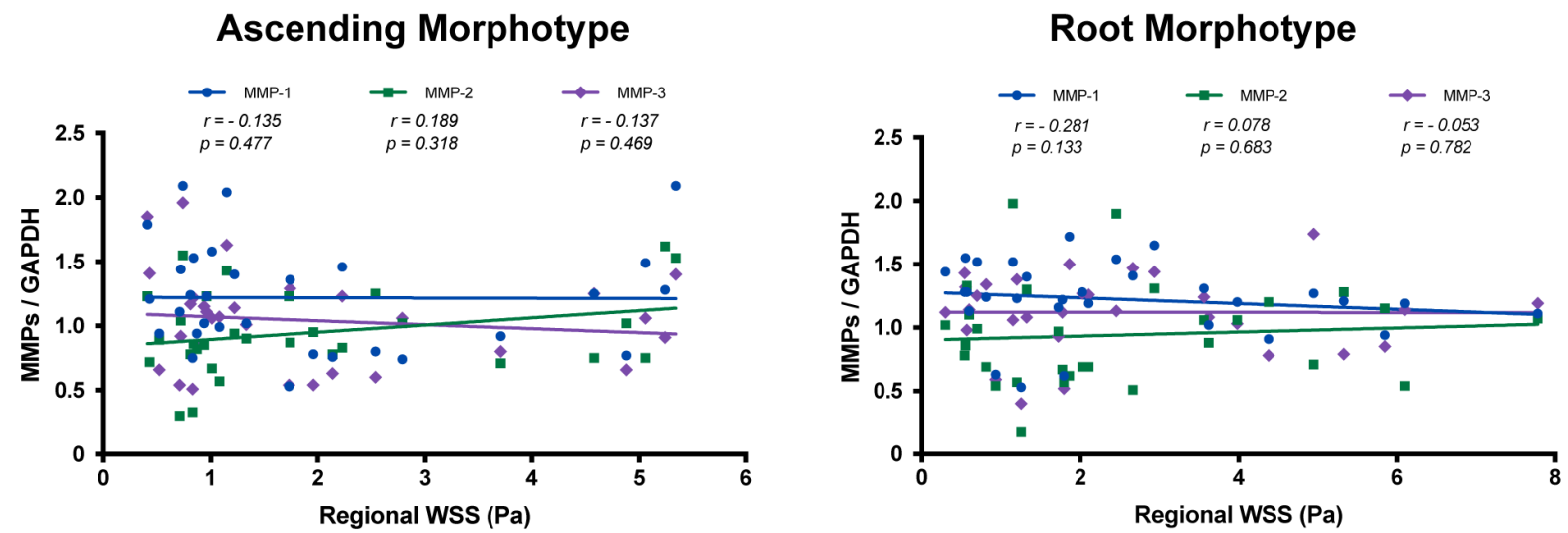

Figure 4. Correlation analysis between regional WSS and MMPs. Correlation coefficients ( $r$ ), P values, and lines of best fit are provided. 
more severe the medial elastin degeneration, the higher the expression of MMPs."

The reason for this phenomenon might be that there exists a competitive inhibition in expression of different MMP subtypes. In the ascending morphotype, the elevated expression level of MMP-2 may lead to a significantly decreased expression of MMP-1 and -3 in the GC region, giving a misleading impression that MMP-1 and -3 are highly expressed on the LC side. Conversely, the same pattern is probably true in the root morphotype. Tissue inhibitor of metalloproteinases (TIMPs) might be the regulators in the process of competitive inhibition in MMP expression between MMP-2 and MMP-1 and -3 [Troeberg 2002]. It has been revealed that the 2 morphotypes have heterogeneous characteristics in distribution and expression of structural proteins in the aortic medial extracellular matrix [Cotrufo 2005], which correspond with our findings that the increase in MMP subtypes is different between them. Laboratory research that focuses on the reasons for different expression status of MMP isoforms in the 2 morphotypes may give a deep etiological understanding of the heterogeneity of bicuspid aortopathy.

Gene-associated inherent predisposition was considered a background factor underlying this disease [Longobardo 2016]. Aortic root dilation frequently occurs at a younger age in male BAV patients [Basso 2004], providing epidemiological evidence to infer that the root morphotype may be a gene-related disease [Sievers 2016]. In this study, it was revealed that valverelated flow turbulence is also a contributing factor for elastic fiber degeneration in patients with root morphotype [Guzzardi 2015]. In addition, medial elastin degeneration was associated with the 2 pathogenetic factors in patients with ascending morphotype. We did not observe a correlation between regional WSS and MMP expression, implying that MMPs may not act as mediators in the process of aortic ECM degeneration induced by increased WSS. The mechanism of aortic ECM remodeling caused by WSS still needs to be explored in further research.

In the guidelines for the management of patients with thoracic aortic disease [Hiratzka 2016], there is still no difference in the recommended threshold of aortic root/ascending aorta replacement between the ascending and root morphotypes of bicuspid aortopathy. Mounting evidence has demonstrated the multifaceted heterogeneity of bicuspid aortopathy [Michelena 2014]. The significant difference of etiology in BAV patients with or without aortic root dilation indicates that a phenotype-specific threshold may be more reasonable for aortic surgery. However, previous clinical studies [Eleid 2013; Wojnarski 2015] that aimed to evaluate the risk of aortic dissection or rupture of BAV patients did not take into account the heterogeneity of bicuspid aortopathy in the procedure of patient selection. Risk evaluation of aortic adverse events separately performed in ascending and root morphotypes would be beneficial in putting forward more precise treatment strategies.

\section{Limitations}

In this study, we did not include a healthy control group because it had been fully revealed that BAV patients had a significant increased regional WSS compared with healthy volunteers.

\section{ACKNOWLEDGMENTS}

We thank Mansu Jin and Runjie Wei of Shengshi Technology, Co., Ltd. (Hangzhou, China) for providing the postprocessing of magnetic resonance images and Hongping Wang of Shengshi Technology for providing the WSS calculations of ascending aorta. Q. Gao is the chief scientist and a cofounder of Shengshi Technology. The other authors declare no conflicts of interest. This work was funded by the National Natural Science Foundation of China (grant number 81701659).

\section{REFERENCES}

Basso C, Boschello M, Perrone C, et al. An echocardiographic survey of primary school children for bicuspid aortic valve. Am J Cardiol 2004;93:661-663.

Bollache E, Guzzardi DG, Sattari S, et al. Aortic valve-mediated wall shear stress is heterogeneous and predicts regional aortic elastic fiber thinning in bicuspid aortic valve-associated aortopathy. J Thorac Cardiovasc Surg 2018;156:2112-2120.e2.

Cotrufo M, Della Corte A, De Santo LS, et al. Different patterns of extracellular matrix protein expression in the convexity and the concavity of the dilated aorta with bicuspid aortic valve: Preliminary results. J Thorac Cardiovasc Surg 2005;130:504-511.

Eleid MF, Forde I, Edwards WD, et al. Type A aortic dissection in patients with bicuspid aortic valves: Clinical and pathological comparison with tricuspid aortic valves. Heart 2013;99:1668-1674.

Fazel SS, Mallidi HR, Lee RS, et al. The aortopathy of bicuspid aortic valve disease has distinctive patterns and usually involves the transverse aortic arch. J Thorac Cardiovasc Surg 2008;135:901-907.e1-2.

Fedak PW, Verma S, David TE, et al. Clinical and pathophysiological implications of a bicuspid aortic valve. Circulation 2002;106:900-904.

Girdauskas E, Borger MA, Secknus MA, Girdauskas G, Kuntze T. Is aortopathy in bicuspid aortic valve disease a congenital defect or a result of abnormal hemodynamics? A critical reappraisal of a one-sided argument. Eur J Cardiothorac Surg 2011;39:809-814.

Guzzardi DG, Barker AJ, van Ooij P, et al. Valve-related hemodynamics mediate human bicuspid aortopathy: Insights from wall shear stress mapping. J Am Coll Cardiol 2015;66:892-900.

Hiratzka LF, Creager MA, Isselbacher EM, et al. Surgery for aortic dilatation in patients with bicuspid aortic valves: A statement of clarification from the american college of cardiology/american heart association task force on clinical practice guidelines. J Am Coll Cardiol 2016;67:724-731.

Huang TS, Yang GJ, Tang GY. A fast two-dimensional median filtering algorithm. IEEE Trans Acoust Speech Signal Process 1979;27:13-18.

Jackson V, Petrini J, Caidahl K, et al. Bicuspid aortic valve leaflet morphology in relation to aortic root morphology: A study of 300 patients undergoing open-heart surgery. Eur J Cardiothorac Surg 2011;40:e118-e124.

Kang JW, Song HG, Yang DH, et al. Association between bicuspid aortic valve phenotype and patterns of valvular dysfunction and bicuspid aortopathy: Comprehensive evaluation using MDCT and echocardiography. JACC Cardiovasc Imaging 2013;6:150-161.

Kari FA, Fazel SS, Mitchell RS, Fischbein MP, Miller DC. Bicuspid aortic valve configuration and aortopathy pattern might represent different pathophysiologic substrates. J Thorac Cardiovasc Surg 
2012;144:516-517.

Kazhdan M, Hoppe H. Screened poisson surface reconstruction. ACM Trans Graph 2013;32:29.

Kendall A, Koochesfahani M. A method for estimating wall friction in turbulent wall-bounded flows. Exp Fluids 2008;44:773-780.

Li F, Gao Q, Qiao E, et al. Contributing factor of proximal arch dilation in patients with bicuspid aortic valve-Wall shear stress or upward extension of ascending aorta dilation? Heart Surg Forum 2020;23:E435-E440.

Longobardo L, Jain R, Carerj S, Zito C, Khandheria BK. Bicuspid aortic valve: Unlocking the morphogenetic puzzle. Am J Med 2016;129:796-805.

Michelena HI, Prakash SK, Della Corte A, et al. Bicuspid aortic valve: Identifying knowledge gaps and rising to the challenge from the international bicuspid aortic valve consortium (BAVCon). Circulation 2014;129:2691-2704.

Nagase H, Woessner JF Jr. Matrix metalloproteinases. J Biol Chem 1999;274:21491-21494.

Otsu N. A threshold selection method from gray level histograms. IEEE Trans Syst Man Cybern 1979;9:62-66.

Rabkin SW. The role matrix metalloproteinases in the production of aortic aneurysm. Prog Mol Biol Transl Sci 2017;147:239-265.

Sbardella D, Fasciglione GF, Gioia M, et al. Human matrix metalloproteinases: An ubiquitarian class of enzymes involved in several pathological processes. Mol Aspects Med 2012;33:119-208.
Schaefer BM, Lewin MB, Stout KK, et al. The bicuspid aortic valve: An integrated phenotypic classification of leaflet morphology and aortic root shape. Heart 2008;94:1634-1638.

Sievers HH, Stierle U, Hachmann RM, Charitos EI. New insights in the association between bicuspid aortic valve phenotype, aortic configuration and valve haemodynamics. Eur J Cardiothorac Surg 2016;49:439-446.

Troeberg L, Tanaka M, Wait R, et al. E. coli expression of timp-4 and comparative kinetic studies with timp-1 and timp-2: Insights into the interactions of timps and matrix metalloproteinase 2 (gelatinase A). Biochemistry 2002;41:15025-15035.

Wang CY, Qi G, Wang HP, et al. Divergence-free smoothing for volumetric piv data. Exp Fluids 2016;57:1-23

Ward C. Clinical significance of the bicuspid aortic valve. Heart 2000;83:81-85.

Wojnarski CM, Svensson LG, Roselli EE, et al. Aortic dissection in patients with bicuspid aortic valve-associated aneurysms. Ann Thorac Surg 2015;100:1666-1673.

Yamashita T, Hayashi T, Tabata T, Hirata KI. Bicuspid aortic valve-associated aortic dilatation-What is the mechanism of bicuspid aortopathy? Circulation 2018;82:2470-2471.

Zhang J, Fan G, Zhao H, et al. Targeted inhibition of focal adhesion kinase attenuates cardiac fibrosis and preserves heart function in adverse cardiac remodeling. Sci Rep 2017;7:43146. 